ARTICLE

Received 26 Jan 2015 | Accepted 27 Mar 2015 | Published 8 May $2015 \quad$ DOl: 10.1038/ncomms8067

\title{
Coupled reversion and stream-hyporheic exchange processes increase environmental persistence of trenbolone metabolites
}

\author{
Adam S. Ward ${ }^{1}$, David M. Cwiertny², Edward P. Kolodziej ${ }^{3,4} \&$ Colleen C. Brehm²
}

Existing regulatory frameworks for aquatic pollutants in the United States are idealized, often lacking mechanisms to account for contaminants characterized by (1) bioactivity of both the parent and transformation products and (2) reversible transformations (that is, metastable products) driven by chemical or physical heterogeneities. Here, we modelled a newly discovered product-to-parent reversion pathway for trenbolone acetate (TBA) metabolites. We show increased exposure to the primary metabolite, $17 \alpha$-trenbolone $(17 \alpha-\mathrm{TBOH})$, and elevated concentrations of the still-bioactive primary photoproduct hydroxylated $17 \alpha-\mathrm{TBOH}$, produced via phototransformation and then converted back to $17 \alpha$-trenbolone in perpetually dark hyporheic zones that exchange continuously with surface water photic zones. The increased persistence equates to a greater potential hazard from parent-product joint bioactivity at locations and times when reversion is a dominant trenbolone fate pathway. Our study highlights uncertainties and vulnerabilities with current paradigms in risk characterization.

\footnotetext{
${ }^{1}$ School of Public and Environmental Affairs, Indiana University, Bloomington, Indiana 47405, USA. ${ }^{2}$ Department of Civil \& Environmental Engineering, The University of lowa, lowa City, lowa 52242, USA. ${ }^{3}$ Interdisciplinary Arts and Sciences, University of Washington, Tacoma, Tacoma, Washington 98402, USA. ${ }^{4}$ Department of Civil \& Environmental Engineering, University of Washington, Seattle, Washington 98195, USA. Correspondence and requests for materials should be addressed to A.S.W. (email: adamward@indiana.edu).
} 
W ith tens of thousands of synthetic chemicals discharged to the environment, it is unrealistic to individually collect comprehensive environmental fate data to accurately define human and ecological health risks. Accordingly, human and ecological risk assessments currently use approaches built upon expectations from the relatively small subset of contaminants whose environmental fates and associated biological risks are well understood. However, the danger implicit in this strategy is that prediction of presumptive biological risks can fail catastrophically for chemical contaminants with reactivities that deviate from expectations. To address this shortfall, computational and numerical modelling efforts can project the environmental fate of chemicals based on a combination of chemical properties and environmental system conditions. Such modelling also can be used to initially screen ecological risks and guide future research efforts such as field sampling. Especially for chemicals of high production volume, widespread environmental occurrence, or unusually high potency and potential toxicity, such efforts are critical to improving assessment of biological risk. As an example, we point to our recent research efforts on the product-to-parent reversion of trenbolone acetate (TBA) metabolites, a previously unidentified chemical process with a poorly understood influence on the environmental fate of these widely used and potent growth promoters ${ }^{1}$.

The use of growth promoting steroid hormones is ubiquitous in the US beef cattle industry, with these pharmaceuticals reducing production costs by up to $7 \%$ and representing $\sim \$ 1$ billion annually in incremental economic value ${ }^{2}$. Administered by ear implantation, TBA is converted to the androgen $17 \beta$-trenbolone, which is far more potent than testosterone and primarily responsible for anabolic effects such as increased weight gain. 17 $\beta$-trenbolone is excreted along with other TBA metabolites including $17 \alpha-\mathrm{TBOH}$ and trendione that are subsequently mobilized in runoff (Fig. 1) ${ }^{3} .17 \alpha-\mathrm{TBOH}$ represents about $95 \%$ of identifiable excreted metabolites by mass ${ }^{4}$, although a number of unidentified TBA metabolites likely exist. Given the typical application rates of TBA to cattle, mass balance calculations predict that thousands of $\mathrm{ng}^{-1}$ are possible in agricultural runoff ${ }^{5,6}$, although concentrations observed in receiving waters are typically far lower when detected ${ }^{7,8}$. Field observations have documented $1,700 \mathrm{ngl}^{-1} 17 \alpha-\mathrm{TBOH}$ in manure lagoons ${ }^{9}$, and $55 \pm 22 \mathrm{ngg}^{-1}$ in solid manure and surface soils ${ }^{10}$. TBA metabolite exposure is problematic for ecosystem health because $17 \alpha-\mathrm{TBOH}$ and $17 \beta-\mathrm{TBOH}$ are potent endocrine disruptors, with exposure to as little as $10-30 \mathrm{ngl}^{-1}$ significantly reducing fecundity, resulting in phenotypic sex reversal in fish, or altering endocrine function ${ }^{11-13}$.

Until recently, rapid photodegradation of TBA metabolites in sunlit surface waters was believed to effectively mitigate their environmental risk $^{14}$. However, we recently reported that the major photoproducts of recognized TBA metabolites, hydroxylated trenbolone species (hereafter hydroxy-17 $\alpha$ $\mathrm{TBOH}$ ), are metastable and able to regenerate their respective parent compounds ${ }^{1}$. These reversible transformations (that is, 'product-to-parent reversion') occur when the rapid forward reaction by direct photolysis (that is, photohydration) in sunlight is countered by slower dehydration reactions when dark (see overview of physical-chemical system in Fig. 1). The relative rates of the forward and reverse reactions ultimately control net photolysis or reversion as a function of both physical characteristics (for example, light exposure) and biogeochemical conditions (for example, $\mathrm{pH}$, temperature).

The first objective of this study is to quantify the impacts of these newly reported reversible reactions on TBA metabolite concentrations in fluvial systems. We designed a series of numerical experiments to highlight key differences between our former (without-reversion) and current (with-reversion) understanding of the environmental fate for TBA metabolites. We hypothesize that the net effect of these processes operating in stream-hyporheic systems is increased persistence of TBA metabolites in the stream system, resulting in enhanced hydraulic transport and higher concentrations. This increased persistence is particularly troublesome for compounds like TBA metabolites

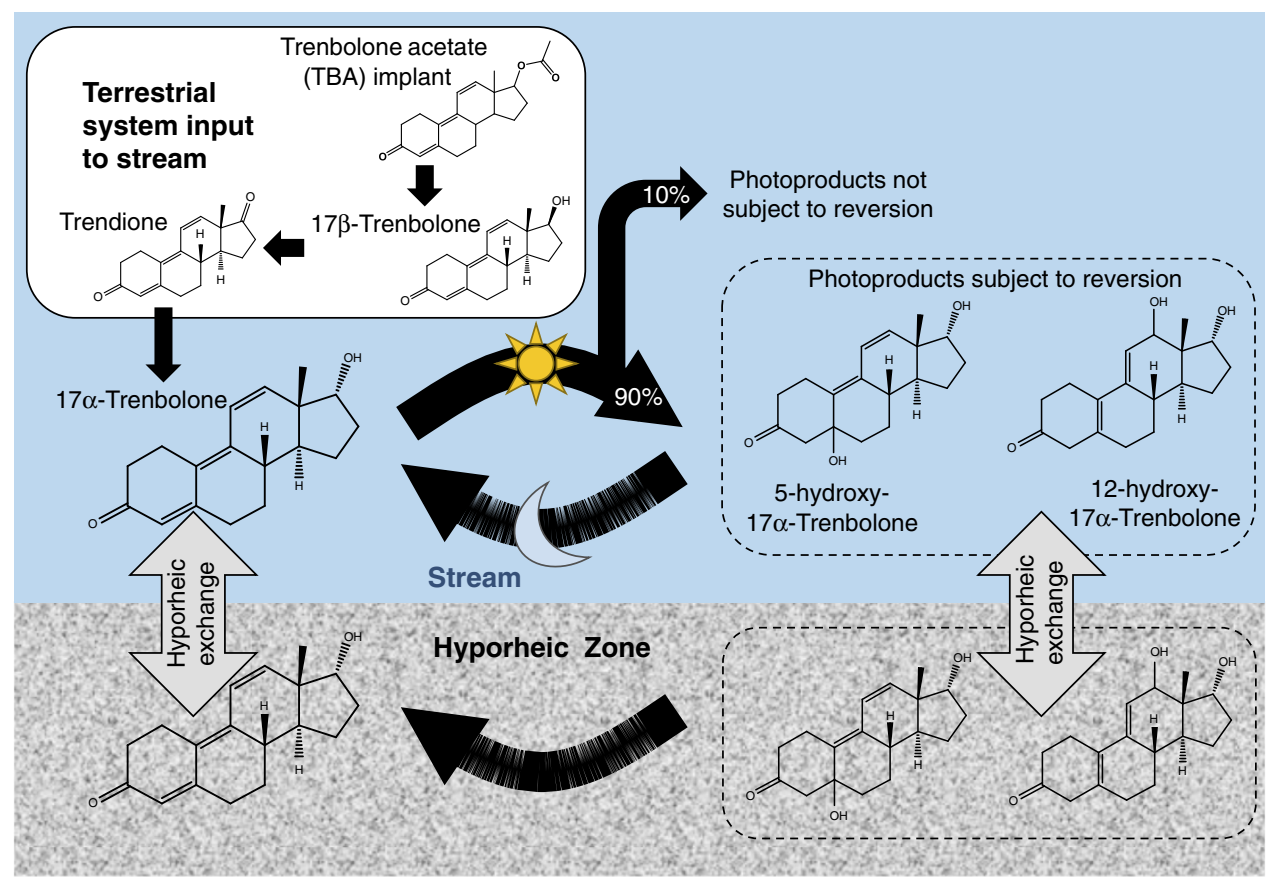

Figure 1 | Overview of interacting physical and chemical systems. Structures of TBA (primary component implanted into cattle), metabolites $17 \beta-\mathrm{TBOH}$ (pharmacologically active androgen), trendione, $17 \alpha-\mathrm{TBOH}$ (primary product excreted to the environment) and $17 \alpha-\mathrm{TBOH}$ photoproducts (5- and 12-hydroxy-17 $\alpha$-TBOH) that are subject to reversion under dark conditions. 
where both the parent species and transformation products retain bioactivity ${ }^{15}$.

Our second objective is to characterize expected joint bioactivity (JBA) arising from the suite of TBA metabolites and their photoproducts, including their spatial and temporal occurrence, partitioning and interactions with hydrological dynamics in stream systems. Accordingly, we calculate JBA withand without-reversion to estimate the risk represented by atypical characteristics like reversible transformation processes, and extend our findings to consider a broader range of risk, wherein JBA is a blend of product and parent concentrations with different relative potencies. This work extends the concept of 'joint persistence' originated by Fenner et al. ${ }^{16-18}$ to account for the range of potencies (that is, bioactivity metrics) expected for parent compounds and related transformation products. We note that, although our simulations are based on TBA metabolites as the pollutant of interest, they are applicable to a range of compounds for which reversible transformations driven by interacting, heterogeneous physical and/or chemical processes occur in the environment.

Our results demonstrate that interactions of primarily physical and chemical processes can yield unexpected transport and fate behaviours, ultimately retaining a substantial fraction of TBA-derived potency in the system over many days. Finally, we generalize model results for cases where differential reactivity controls parent-product partitioning and JBA in the system. Although this modelling effort focuses on an idealized case for the transport and fate of TBA metabolites to better understand the environmental implications of their reversion pathway, results are applicable more generally to any system where heterogeneity in the physical or chemical system affects parent-product fate dynamics. Ultimately, we demonstrate that current assessment approaches remain prone to unintended consequences because they are ill-equipped for pollutants with atypical transformation pathways and they cannot accurately predict how heterogeneous physical and chemical environments control JBA.

\section{Results}

Reversion increases $17 \alpha-\mathrm{TBOH}$ persistence in stream networks. The numerical simulations demonstrate that reversion cycling increases in-stream and hyporheic $17 \alpha-\mathrm{TBOH}$ concentrations compared with the without-reversion case throughout the stream network (Fig. 2). Spatial profiles for cases with- and withoutreversion are shown for both $17 \alpha-\mathrm{TBOH}$ and hydroxy-17 $\alpha$ TBOH throughout a 24-hr cycle in Fig. 2 (see also Supplementary Movie 1, Supplementary Fig. 1). The spatial and temporal scales to achieve removal of peak concentrations during a 24-hour cycle as the management target of interest vary between cases (maximum of shaded 24-hour range for $17 \alpha-\mathrm{TBOH}$; Fig. 3). Advective travel times to achieve $50 \%$ removal of input concentrations are 14.4 and $13.8 \mathrm{~h}(38.0$ and $36.2 \mathrm{~km})$ for the cases with- and without-reversion, respectively. Times to $90 \%$ removal are 287.4 and $16.2 \mathrm{~h}(757.4$ and $42.7 \mathrm{~km})$ for with- and withoutreversion cases, respectively, and the disparity grows to 406.9 and $25.9 \mathrm{~h}(1,072.4$ and $68.2 \mathrm{~km})$ to achieve $99 \%$ removal (linear extrapolation beyond the model domain for the with-reversion case). In contrast, timescales for the forward reaction in idealized laboratory studies were reported as 1.1 to $2.5 \mathrm{~h}$. In our simulations, the complexity of the fluvial environment-particularly the perpetually-dark hyporheic zone where reversion processes would dominate even during periods of light-results in the increased persistence of $17 \alpha-\mathrm{TBOH}$ in the stream.

Patterns of $17 \alpha-\mathrm{TBOH}$ are spatially and temporally variable in the stream network. At night, the inflowing $17 \alpha-\mathrm{TBOH}$ is primarily advected in the stream channel. Hyporheic exchange decreases maximum in-stream concentrations of $17 \alpha-\mathrm{TBOH}$ due to exchange between the relatively high concentration stream water and the low concentration hyporheic water in both withand without-reversion cases (Fig. 3(1)). The decrease is more pronounced in the without-reversion case, where concentration gradients between the stream and hyporheic zone are steeper (hyporheic reversion to $17 \alpha-\mathrm{TBOH}$ decreases this gradient in the with-reversion case).

The results in Fig. 3 provide data that could be used to estimate both acute and chronic exposure, best characterized by the maximum and mean concentrations, respectively. After $12 \mathrm{~h}$ of advection, maximum in-stream concentrations are 77.1 and $86.3 \%$ of the input concentration for the without-reversion and with-reversion cases, respectively. When forward photolysis rates exceed reversion rates (during periods of light), rapid decreases of in-stream $17 \alpha-\mathrm{TBOH}$ concentrations occur in both cases (Fig. 3(2)). In-stream $24 \mathrm{~h}$ minimum values are dominated by the forward photolysis reaction (Fig. 3(3)), achieving initial removals of 50 and $90 \%$ at 0.34 and $1.20 \mathrm{~h}$ for the withoutreversion case, and 0.35 and $1.24 \mathrm{~h}$ for the with-reversion case. Removal of $99 \%$ of the input $17 \alpha-\mathrm{TBOH}$ is achieved for the without-reversion case after $25.9 \mathrm{~h}$ (Fig. 3(4)), while daily peak $17 \alpha-\mathrm{TBOH}$ concentrations of 30.4 and $40.9 \%$ persist in the stream and hyporheic zone, respectively (Fig. 3(5)). Finally, beyond $38 \mathrm{~km}$ in the network, hyporheic zone concentrations of $17 \alpha-\mathrm{TBOH}$ are higher than in-stream concentrations throughout a complete $24 \mathrm{~h}$ cycle (Fig. 3(6)). Upstream of this location, hyporheic concentrations oscillate between being higher and lower than in-stream concentrations. This location defines a transition point from an upstream source-dominated reach to a downstream reversion-dominated reach.

The transition location is primarily set by in-stream advection and photoperiod, specifically the distance $17 \alpha-\mathrm{TBOH}$ is transported during periods where the reversion rate exceeds the photolysis rate. Longer photoperiods or slower advective velocities move this location closer to the source. In these cases, the partitioning between the stream and hyporheic zone, responsible for the declining maximum concentrations in-stream (Figs 2-4), decreases in importance.

Hyporheic zones exhibit time-variable source-sink behaviour. Transport and transformation in the stream interact with storage and reversion in the hyporheic zone along the stream, leading to extended resupply of $17 \alpha-\mathrm{TBOH}$ to the stream and elevated in-stream concentrations (Figs 2 and 3; Supplementary Movie 1). The hyporheic zone acts as a net source of $17 \alpha-\mathrm{TBOH}$ to the stream when hyporheic concentrations exceed those in-stream (that is, stream time series above hyporheic time series), and a net sink at times when $17 \alpha-\mathrm{TBOH}$ concentrations are lower in the hyporheic zone than in the stream (that is, stream time series below hyporheic time series; Fig. 4 and Supplementary Fig. 2). Net source or sink behaviour is defined by the dynamic development of concentration gradients between the stream and hyporheic zone, and the magnitude defined by the exchange rate $(\alpha)$ and relative sizes of the stream and hyporheic zone (commonly $A_{\mathrm{s}} / A$ ). This dynamic source-sink characteristic is in contrast to definitions of source and sink used for chemical systems without considering product-to-parent reversion, where sinks often indicate irreversible loss, and sources indicate new mass (as opposed to reverted mass) entering the system.

The input $17 \alpha-\mathrm{TBOH}$ is primarily advected downstream of the input plateau concentration during periods of darkness (Fig. 4). The exchange of water between the stream and hyporheic zones results in a hyporheic concentration always trending towards the in-stream concentrations, but never reaching equilibrium (Fig. 4). The characteristic timescale of exchange is longer than the 

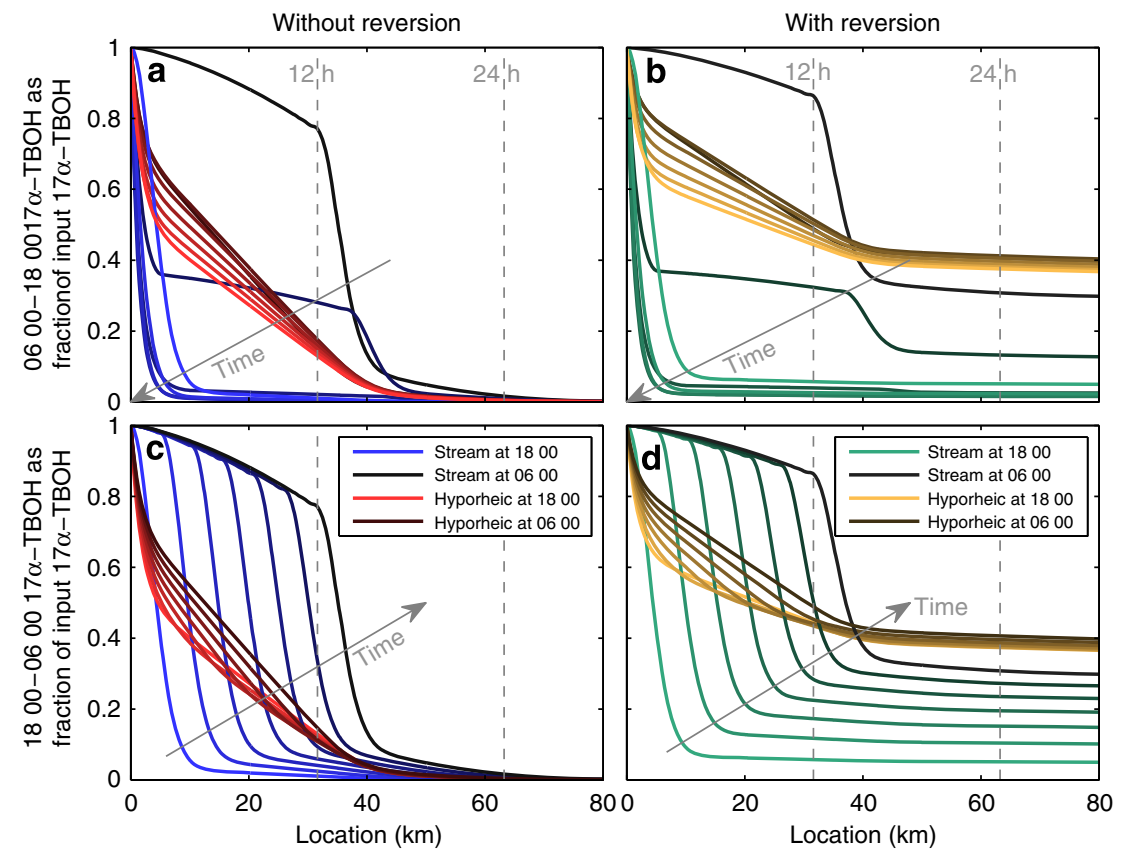

Figure 2 | Spatial profiles of $\mathbf{1 7} \alpha-$ TBOH through a 24-hour cycle. Spatial profiles for $17 \alpha-\mathrm{TBOH}$ without (left column) and with (right column) product-to-parent reversion. Profiles are shown through a typical 24-hour cycle divided into periods of daylight (top row, 0600-1800 hours) and darkness (bottom row, 1,800-0,600 hours), with individual profiles at 2-hour intervals. Vertical dashed lines indicate distances for 12 and $24 \mathrm{~h}$ of downstream advection in the stream channel. Concentration profiles reflect the interaction of physical transport processes (primarily advection, hyporheic exchange) with chemical processes (time-variable photolysis, persistent reversion). The stream profile is dominated by advection of the input concetrations of $17 \alpha-\mathrm{TBOH}$ during periods of darkness (bottom row) and by photolysis during periods of light (top row). Reversion of hydroxy-17 $\alpha-\mathrm{TBOH}$ to $17 \alpha-\mathrm{TBOH}$ increases hyporheic concentrations throughout the domain, particularly at distances downstream of about $10 \mathrm{~km}$.
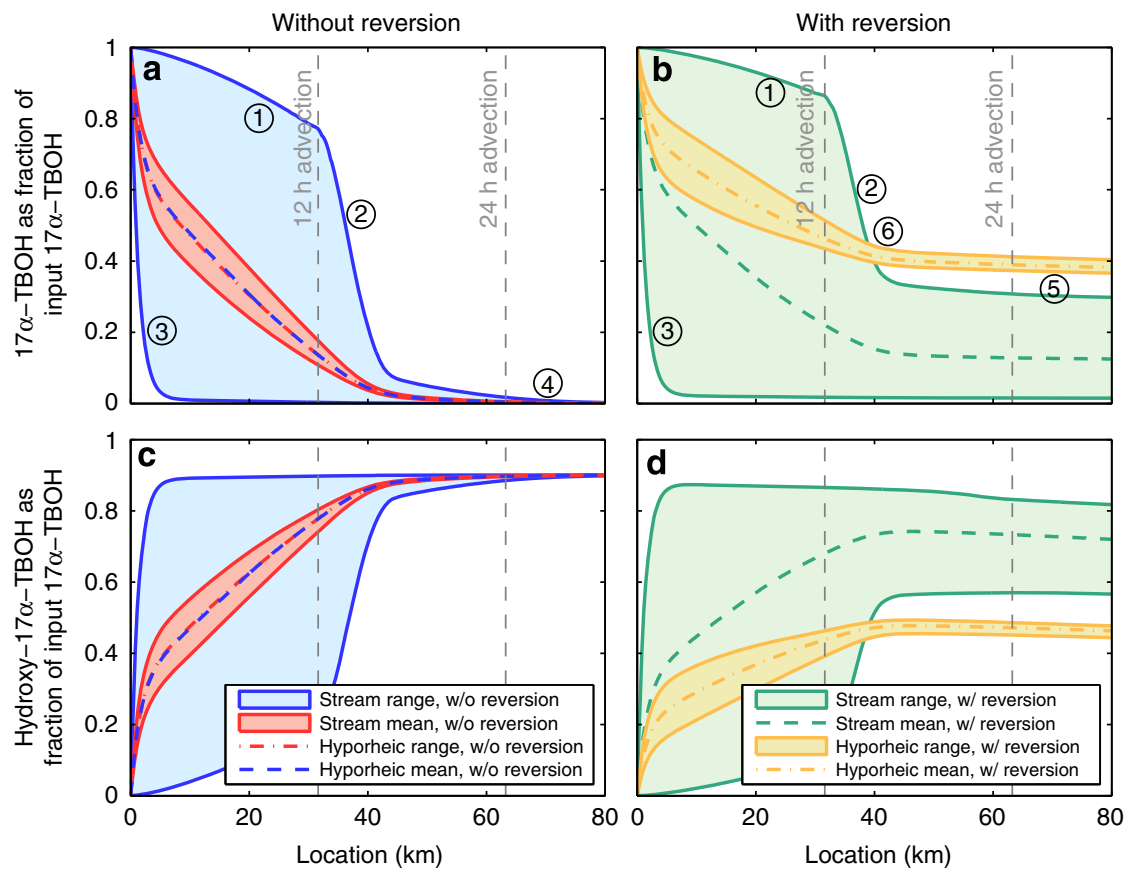

Figure 3 | Twenty-four-hour average, maximum and minimum 17 $\alpha$-TBOH and hydroxy-17 $\alpha$-TBOH concentrations. Twenty-four-hour average concentrations (dashed lines) and ranges (shaded regions) for $17 \alpha-\mathrm{TBOH}$ and hydroxy-17 $\alpha-\mathrm{TBOH}$ in the stream-hyporheic system through a typical 24-hour cycle at dynamic steady state. Vertical dashed lines indicate distances for 12 and $24 \mathrm{~h}$ of downstream advection in the stream channel. Labels indicate (1) advection of input concentration in-stream during period of darkness; (2) rapid photolysis of $17 \alpha-\mathrm{TBOH}$ advected during night time period, decay is not exponential because the forward reaction rate is time-variable with solar loading; (3) rapid photolysis of $17 \alpha-\mathrm{TBOH}$ input during daylight period; (4) $99 \%$ removal of $17 \alpha-\mathrm{TBOH}$ achieved after $25.9 \mathrm{~h}$; (5) equilibrium of photolysis and reversion, linear decay due to partitioning of $10 \%$ of forward reaction of $17 \alpha-\mathrm{TBOH}$ to non-revertible products; and (6) hyporheic zone has only a source function downstream of $38 \mathrm{~km}$. 

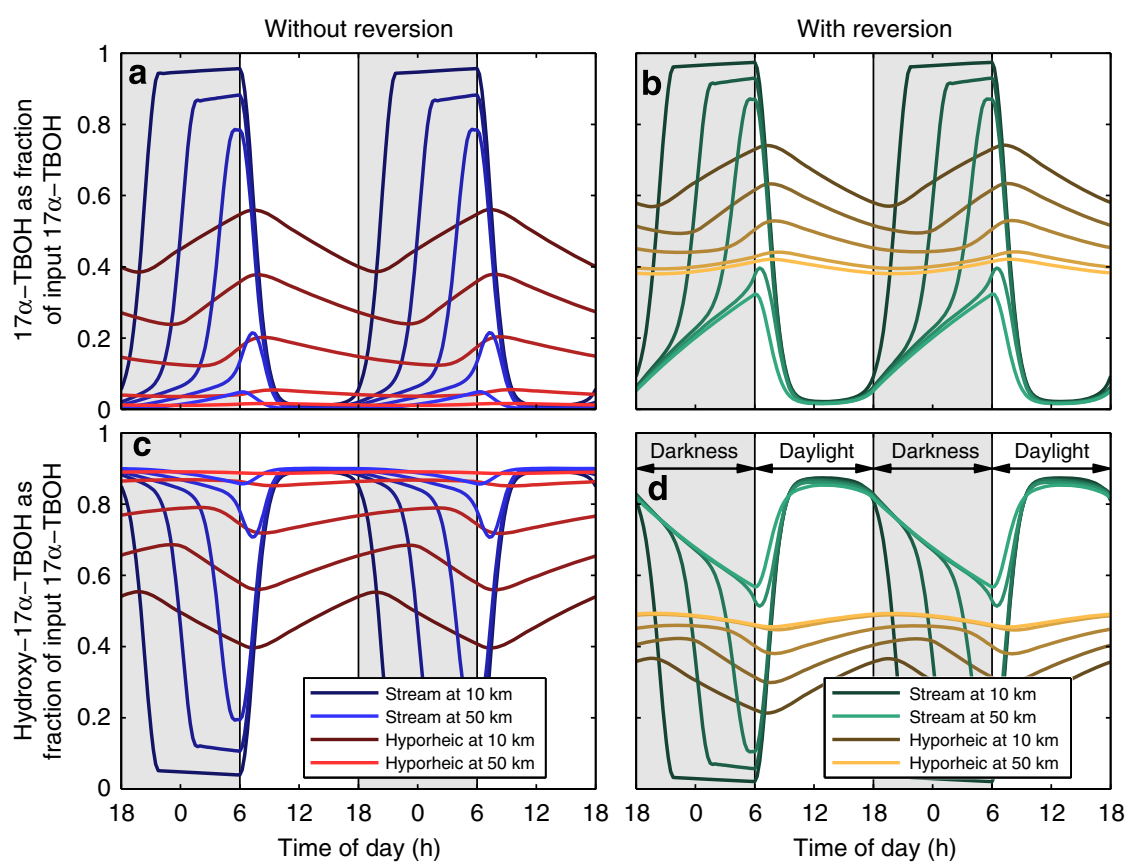

Figure 4 | Time series of in-stream and hyporheic 17 $\alpha$-TBOH and hydroxy-17 $\alpha$-TBOH concentration at selected spatial locations. Time series of stream and hyporheic concentrations for $17 \alpha-\mathrm{TBOH}$ without (left column) and with (right-column) product-to-parent reversion at selected locations. Two typical $24 \mathrm{~h}$ cycles are presented for each spatial location. Grey and white shaded regions indicate periods of darkness (reversion dominance in the stream) and daylight (photolysis dominance in the stream), respectively. For cases without-reversion, hyporheic zones alternate between net sources of $17 \alpha-\mathrm{TBOH}$ to the stream. The hyporheic zone is a net source of $17 \alpha-\mathrm{TBOH}$ when stream concentration is less than hyporheic concentration (that is, during daylight periods when photolysis dominates in the stream). Hyporheic zones are a net sink for in-stream $17 \alpha-\mathrm{TBOH}$ when the stream concentration is greater than the hyporheic concentration (typically during periods of darkness). Cases with and without-reversion have similar source-sink behaviour for the first $38 \mathrm{~km}$ of transport, but hyporheic zones become persistent sources of $17 \alpha-\mathrm{TBOH}$ to the stream beyond this location. In these downstream locations, hyporheic reversion is able to increase in-stream concentrations to nearly $40 \%$ of their input concentration in some locations. The diminishing night time plateau in the downstream direction represents decreased influence of the night time input signal to the diel pattern at a spatial location.

timescale driving in-channel dynamics (that is, the photoperiod). Exchange of water between the stream and hyporheic zone reduces the amplitude of the diel in-stream concentration time series. Similar hyporheic buffering for stream temperatures varying on a diel basis has been broadly reported. Temporary storage of $17 \alpha-\mathrm{TBOH}$ advected downstream during periods of darkness, exchanged into the hyporheic zone, stored temporarily in this domain with no photolysis, and then slowly released back to the stream increases in-stream minimum concentrations of $17 \alpha-\mathrm{TBOH}$. Similarly, the inverse processes occur in daylight and decrease in-stream maximum concentrations. Hyporheic buffering of in-stream concentrations occurs throughout the model domain for both the with- and without-reversion cases. For the case without-reversion, the timing and magnitude of the net source and sink behaviour are solely a reflection of the characteristic timescale of hyporheic exchange relative to the timescale of diel in-stream fluctuations. Large exchange rates and small hyporheic zones cause hyporheic $17 \alpha-\mathrm{TBOH}$ concentrations to converge on dynamics identical to those in the stream alone, reducing the buffering effect, while the opposite occurs for the inverse case. For a smaller hyporheic zone and/or faster exchange rate, the stream and hyporheic zone would more closely track in their timeseries. At one extreme, the hyporehic zone would be instantaneously well-mixed with the stream and have minimal impact on the $17 \alpha-\mathrm{TBOH}$ timeseries at a location, due to either a small hyporheic zone or extremely rapid exchanges with the stream. Conversely, a low exchange rate would functionally decouple the stream and hyporheic zone.

For the case with reversion, the exchange processes described above interact with slowly increasing hyporheic $17 \alpha-\mathrm{TBOH}$ concentrations as product-to-parent reversion occurs. After $24 \mathrm{~h}$ of transport, daily average in-stream $17 \alpha-\mathrm{TBOH}$ concentrations are 12.8 and $0.4 \%$ of the input concentrations for the with- and without-reversion cases. Throughout the model domain, particularly the reversion-dominated reach, reversion maintains higher $17 \alpha-\mathrm{TBOH}$ concentrations in the stream and hyporheic zone. Increased hyporheic concentrations reduce the net sink function of the hyporheic zone, proportionally increasing its source function (Fig. 3). The magnitude of the net source function is controlled by the rate of $17 \alpha-\mathrm{TBOH}$ regeneration relative to the stream-hyporheic zone exchange rate. The source function is minimized wherever the exchange rate removes $17 \alpha-\mathrm{TBOH}$ faster than it can be regenerated. In contrast, the without-reversion case loses $10 \%$ of the total simulated mass (during the forward photolysis reaction) and then conservatively transports the remaining $90 \%$ as hydroxy-17 $\alpha-\mathrm{TBOH}$.

Although we expect that other attenuation processes affect transport and fate of $17 \alpha-\mathrm{TBOH}$ and hydroxy-17 $\alpha-\mathrm{TBOH}$, sufficient data characterizing such processes are not available for both species and thus we omitted them from our simulations. Nevertheless, expected behaviour can be described. Reversible sorption-desorption processes would create additional time lags in the temporary storage of $17 \alpha-\mathrm{TBOH}$, increasing buffering of in-stream concentrations (assuming minimal photolysis in the sorbed phase, primarily within the hyporheic zone). Sorption would also represent an irreversible removal of dissolved mass for a permanently bound fraction of the sorbed species. Biotransformation of $17 \alpha-\mathrm{TBOH}$ is expected to compete with reversion and reduce concentrations in the stream and/or hyporheic zone. We note, however, that such competing reactions are already 
integrated into our simulation, with a constant reversion rate and time-variable photolysis rate interacting dynamically though time. On the basis of experimental yields of photoproducts reported by $\mathrm{Qu}$ et al. ${ }^{1}$, our model partitions $10 \%$ of the photoproducts to non-bioactive species incapable of reversion (for example, species with a substantially modified structure ${ }^{15}$ ). This represents a continuous loss of mass over time as product and parent continue to cycle. Additional competing reactions would be expected to simply increase the magnitude of this loss term. If all loss processes are considered first order, the net sum of their reaction rate coefficients would dictate the behaviour of the system. For example, a hyporheic zone with a biotransformation rate for $17 \alpha-\mathrm{TBOH}$ larger than its reversion rate would exhibit increased sink behaviour. Periods of net source behaviour are expected to persist even in this case. Finally, characterization of non-linear interactions (for example, those associated with differing behaviour of sorbed and dissolved compounds in the hyporheic zone) is generally lacking and would require increased model complexity.

Yield and bioactivity partitioning control ecological risk. One benefit of our modelling approach is that we can explore fate and risk scenarios that might otherwise be too difficult to assess via traditional approaches wholly reliant on demanding and expensive analytical measurements. For example, a scenario that complicates risk management for TBA metabolites is that their photoproducts seem to retain some aspects of bioactivity distinct from their parent metabolites ${ }^{15}$. Thus, confining ecological risk considerations only to the known bioactivity of $17 \alpha-\mathrm{TBOH}$ would underestimate the ecological hazard posed by the mixture of parent and product bioactivity. This case is not just limited to TBA metabolites, as a growing number of studies demonstrate increased JBA, or total combined bioactivity, attributed to mixtures of parent compounds, metabolites and transformation products. In fact, summed mixture persistence in a network has been termed 'joint persistence' where several reactions occur in series to account for the summed persistence of all related compounds ${ }^{16-18}$. Inclusion of bioactivity metrics in a similar framework is a logical extension of this concept.

We note that the concept of JBA is beginning to be addressed using regulatory concepts such as 'predicted no-effect concentrations (PNECs)' for which mixture bioactivity is the key metric of interest rather than any single concentration of individual constituents ${ }^{19,20}$. Indeed, it is probably most logical to move away from regulatory approaches structured around individual constituents in general, and towards biologically driven end points such as those structured within adverse outcome pathways $^{21}$. In some cases, accounting for JBA causes mixtures to exceed PNECs that would not otherwise be met if only the parent compound were considered ${ }^{22}$. Trenbolone metabolites are ideal examples to illustrate mixture bioactivity effects because their product-to-parent reversion dynamics will be key contributors to JBA in affected watersheds. Further, our modelling approach enables us to quantify JBA for $17 \alpha-\mathrm{TBOH}$ and its primary photoproduct, hydroxy-17 $\alpha-\mathrm{TBOH}$ in a streamhyporheic system at a resolution that is not possible from laboratory experiments or field data alone.

We used our model to consider five representative scenarios where bioactivity was apportioned to $17 \alpha-\mathrm{TBOH}$ and hydroxy-17 $\alpha-\mathrm{TBOH}$ through a range of relative potencies (see
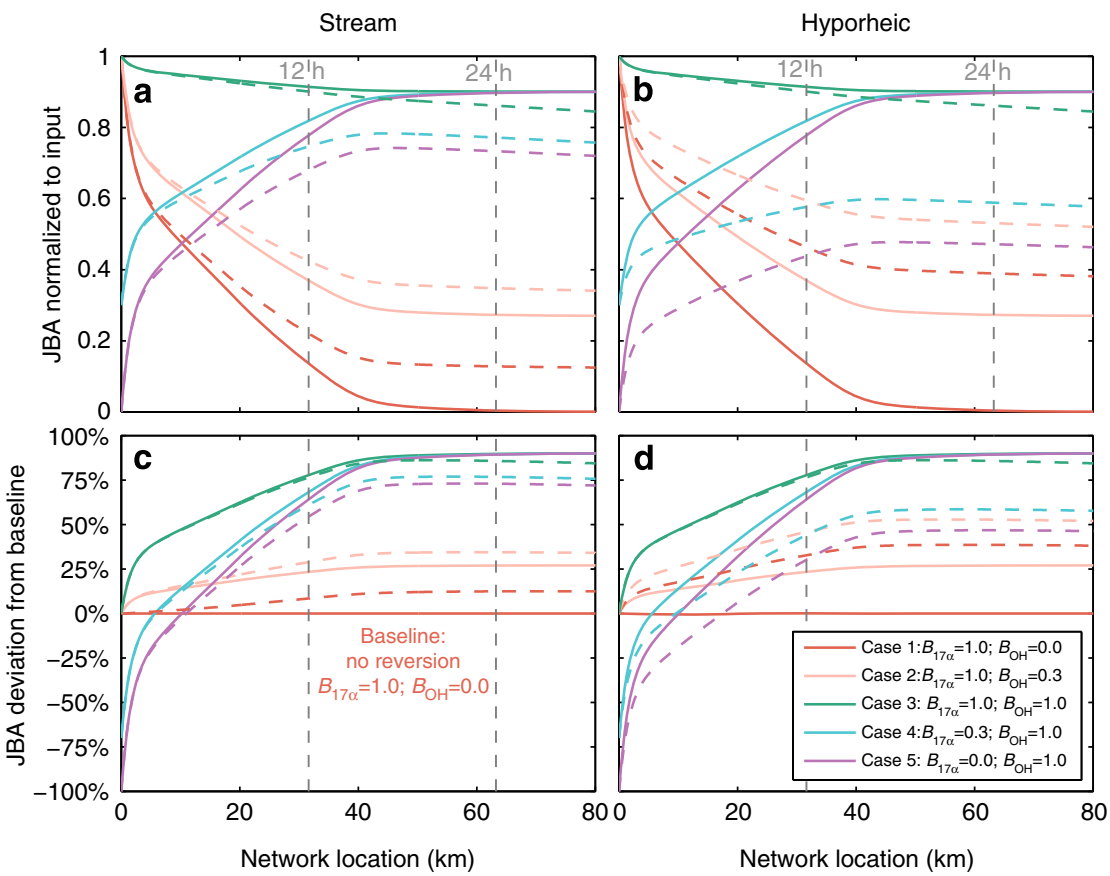

Figure 5 | Joint bioactivity in the stream and hyporehic zone. In-stream joint bioactivity from simulations of $17 \alpha-\mathrm{TBOH}$ and hydroxy-17 $\alpha-\mathrm{TBOH}$, calculated by scaling in-stream concentrations by bioactivity coefficients and summing values for both species (top row). Representative cases were calculated with and without-reversion for a range of relative bioactivities, including: Case 1: parent only, Case 2: parent more bioactive than product, Case 3: equal bioactivity, Case 4: product more bioactive than parent and Case 5: product only. Joint bioactivity (JBA) is calculated by scaling concentrations by bioactivity coefficients, and presented as JBA in the stream (left column) and hyporheic zone (right column). In addition, we present the deviation from the baseline conditions where only $17 \alpha-\mathrm{TBOH}$ is bioactive and no reversion occurs, the de facto regulatory assumption (bottom row). To demonstrate how the inclusion of increased complexity in chemical processes and bioactive metabolites changes JBA relative to currently regulatory assumptions (only $17 \alpha-$ $\mathrm{TBOH}$ is bioactive, no reversion; Case 1 solid lines). In simulations with reversion, the linear decay of bioactivity in the systems with reversion is due to the continued partitioning of $10 \%$ of the forward reaction to non-revertible, non-bioactive products. Vertical dashed lines indicate distances for 12 and $24 \mathrm{~h}$ of downstream advection in the stream channel. 
Methods section). Bioactivity coefficients include subscript ' $17 \alpha$ ' for $17 \alpha-\mathrm{TBOH}$ and ' $\mathrm{OH}$ ' for hydroxy-17 $\alpha-\mathrm{TBOH}$. The subscripts ' $w$ ' and 'w/o' denote cases with or without product-to-parent reversion, respectively. As a baseline for comparison (Case $1_{\mathrm{w} / \mathrm{o}}$ ), we set a JBA value of 1.0 to represent the bioactivity expected solely by input of $17 \alpha-\mathrm{TBOH}$ at the upstream end of the model domain, without considering reversion. This case is considered the baseline for risk assessment because it represents the current regulatory approach to $17 \alpha-\mathrm{TBOH}$ fate in the United States (that is, no reversion, no bioactivity attributed to transformation products). For all subsequent cases, we present both absolute JBA and the change in JBA relative to the baseline case (Fig. 5).

For the baseline scenario (Case $1_{\mathrm{w} / \mathrm{o}} ; B_{17 \alpha}=1 ; B_{\mathrm{OH}}=0$ ), bioactivity is equal to the concentration of $17 \alpha-\mathrm{TBOH}$, rapidly decreasing in the stream and hyporheic zone upon $17 \alpha-\mathrm{TBOH}$ transformation. Daily average JBA in both the stream and hyporheic zone is reduced by $99 \%$ at a distance of about $53 \mathrm{~km}$ in the network. We take this network location as a key point for comparison between scenarios relative to the baseline. Withoutreversion cases approach a plateau beyond this distance, where only dilution by inflows reduces in-stream JBA. For Case $1_{\mathrm{w}}$, we found increases in in-stream and hyporheic JBA by 12 and 39\% above the baseline at $53 \mathrm{~km}$, respectively. Beyond this network location, all cases with reversion exhibited a persistent JBA fraction in the network, only decreasing by about $1.3 \%$ of input JBA per $100 \mathrm{~km}$ due to the $10 \%$ partitioning to irrevertible, non-bioactive products during each photolysis-reversion cycle. This rate applies to the all source-dominated cases that include reversion.

For Case $2_{\mathrm{w} / \mathrm{o}}\left(B_{17 \alpha}=1 ; B_{\mathrm{OH}}=0.3\right)$, where the primary transformation product hydroxy-17 $\alpha-\mathrm{TBOH}$ retains partial bioactivity, JBA in the stream and hyporheic zone are increased over the baseline scenario by $27 \%$ at $53 \mathrm{~km}$. For Case $2_{\mathrm{w}}$, increases in JBA are 34 and $53 \%$ for the stream and hyporheic zone, respectively. For any cases where $B_{17 \alpha}>B_{\mathrm{OH}}$, concave-up patterns of JBA will be produced in the network, indicating decreasing exposure in the downstream direction and equilibrium favours the less-active form, hydroxy-17 $\alpha-\mathrm{TBOH}$.

For Case $3_{\mathrm{w} / \mathrm{o}}\left(B_{17 \alpha}=1 ; B_{\mathrm{OH}}=1\right)$, in-stream and hyporheic JBA are about $89 \%$ larger than the baseline value at $53 \mathrm{~km}$ because the product is substantially more potent than in Case 2. Despite the fact that parent and product are equipotent, input JBA is not perfectly conserved because the stream and hyporheic zone must come into equilibrium. Declines in JBA along the stream reflect the unequal distribution of steroid mass between the stream and hyporheic zone. Elevated JBA levels persist indefinitely in these simulations and are only reduced by competing physical or chemical attenuation processes (for example, dilution by inflow, sorption, biotransformation) in the network. For Case $3_{w / o}$, the $10 \%$ partitioning to non-bioactive products occurs only once (that is, no mass undergoes photolysis more than one time), resulting in a constant exposure of JBA through the network after this stream-hyporheic partitioning (horizontal lines in Fig. 5). For Case $3_{\mathrm{w} / \mathrm{o}}$, in-stream and hyporheic JBA are $86 \%$ larger than baseline values at $53 \mathrm{~km}$. The slight reduction in JBA compared with Case 3a reflects reverted $17 \alpha-\mathrm{TBOH}$ being photolysed and including $10 \%$ conversion to stable products over multiple cycles.

Cases 4 and 5 reflect systems where product potencies exceed parent potency $\left(B_{17 \alpha}<B_{\mathrm{OH}}\right)$. In each case the input JBA is less than the baseline in relative terms because of the lower potency parent, but JBA rapidly increases in the network as the forward reaction proceeds, exhibiting a characteristic concave-down profile. For Case $4_{\mathrm{w} / \mathrm{o}}\left(B_{17 \alpha}=0.3 ; B_{\mathrm{OH}}=1\right)$, in-stream JBA at $53 \mathrm{~km}$ is increased by $88 \%$ compared with baseline conditions in both the stream and hyporheic zone. For Case $4_{w}$, increases in
JBA over the baseline case in the stream and hyporheic zone are 77 and $58 \%$ at $53 \mathrm{~km}$, respectively. For Case $5_{\mathrm{w} / \mathrm{o}}\left(B_{17 \alpha}=0\right.$; $\left.B_{\mathrm{OH}}=1\right)$, JBA in the stream and hyporheic zone is $88 \%$ higher than baseline values at $53.3 \mathrm{~km}$. For Case $5_{w}$, increases over baseline are 73 and $47 \%$ at $53 \mathrm{~km}$. For cases with $B_{17 \alpha}<B_{\mathrm{OH}}$, representing formation of relatively more potent products, it is not surprising that environmental risk assessment is especially challenging because the location of maximum JBA exposure is not proximal to the release, which necessitates analysis of hydraulic retention times and reaction times. Thus, for cases where products retain some bioactivity, especially where product bioactivity arises from distinct pathways (for example, interacts with a different receptor, different biochemical pathway), it is reasonable to expect maximum impacts at locations distant from contaminant sources.

\section{Discussion}

We found that hyporheic exchange and reversion processes increase downstream JBA by $34 \%$ in the stream and $53 \%$ in the hyporheic zone (Case $2_{\mathrm{w}}$ ) compared with those estimated by considering only the irreversible forward reaction (Case $1_{\mathrm{w} / \mathrm{o}}$ ). At the time of publication, reliable field sampling and measurement protocols for TBA metabolites and photoproducts have not been established. Thus, the outcomes detailed here are projected based on state-of-the-science laboratory results and numerical models. Compared with a baseline case in which mass is assumed to be fully removed by photolysis occurring rapidly in the stream, these sustained concentrations pose a significant, additional hazard. At a minimum, we believe these results justify the need for revisiting regulatory strategies and considering the use of mixture based PNECs. For endocrine disrupting compounds in general, where source concentrations have been documented at thousands of $\mathrm{ng} \mathrm{l}^{-1}$ and impacts demonstrated for tens of $\mathrm{ng} \mathrm{l}^{-1}$ or less, these increased concentrations may be ecologically relevant.

This modelling also provides a clear demonstration that simplified fate models, such as half-life, are most applicable only proximal to the source. To estimate downstream concentrations or JBA accurately, a new, more complete paradigm is needed. This approach may need to be compound specific, and it must explicitly consider the dominant interacting physical and chemical processes, including formation of transformation products, that govern transport and fate outcomes to avoid underestimation of environmental risk. This issue would be particularly acute for bioactive species with moderate-to-high persistence or potent species known to yield bioactive products, as these compounds may lack sufficient attenuation mechanisms to reduce JBA and associated risk in environmental systems.

For compounds where even trace concentrations are ecologically relevant, current risk assessment paradigms are particularly vulnerable to atypical processes such as the reversible transformation driven by physical and/or biogeochemical heterogeneities. For the TBA metabolite simulations presented here, if a PNEC limit is not achieved within the source-dominated reach where rapid decay of JBA dominates, subsequent transport in the reversion-dominated reach will maintain concentrations above the PNEC threshold and JBA will persist at unsafe levels. In systems characterized by product-to-parent reversion, effective removal in the source-dominated reach is critical if a reaction process, such as rapid photo- or biotransformation, dominates expected reductions in environmental risk. Management targeting the source of TBA metabolites or their removal within the sourcedominated reach is therefore important to reduce any impact on fluvial ecosystems, rather than relying on naturally occurring attenuation processes farther downstream in the network.

Model simulations demonstrate increased persistence for $17 \alpha-\mathrm{TBOH}$ and associated JBA in stream systems, where the 
hyporheic zone becomes a hot-spot for product-to-parent reversion and unexpectedly acts as an important diffuse source of bioactive parent to surface waters. Although $17 \alpha-\mathrm{TBOH}$ dynamics are similar in the source-dominated reach for withand without-reversion cases, where $\sim 60 \%$ of removal occurs, reversion subsequently increases removal timescales by a full order of magnitude in the reversion-dominated reach. Finally, depending on relative parent-product potency, which admittedly remains uncertain for most classes of environmental pollutants, we demonstrate the substantial divergence of bioactivity predictions with- and without-reversion, particularly with respect to defining long-term contaminant persistence in the stream. Current regulatory paradigms that focus on individual species fail to address joint persistence and JBA in the environment. Furthermore, those based on representative timescales of an individual reaction are insufficient to characterize ecosystem exposure for chemical systems characterized by product-toparent reversion and reaction rates driven by heterogeneity in environmental systems (for example, differential reactivity in perpetually dark hyporheic zones versus dynamic forward reactions in streams).

The key issue highlighted by this study is demonstrating the potential implications that an atypical or unexpected contaminant characteristic (for example, arising from either environmental reactivity, fate or bioactivity) has on the accuracy of environmental risk assessment. Our simulations demonstrate that conservation of bioactivity between species, especially in systems where physical and chemical heterogeneity interact to control partitioning of parent and transformation products, can represent a critical yet underappreciated component of JBA and subsequent environmental risk. Relative to parent compounds, bioactivity in transformation products and mixtures remains an emerging area of research and is an integral aspect of any transition to systems analysis driven by biological endpoints.

Relative environmental risk is a function of yield, potency and persistence. During assessment, unexpectedly high values for any of these parameters represent opportunities for significant underestimation of environmental risk ${ }^{23}$. Accurate understanding of the relationships between these key controls, particularly those efforts that develop insight into potency across biological endpoints, would build more confidence in the effectiveness of any particular environmental risk assessment. Similarly, we demonstrate that, for instances of atypical reaction characteristics and pathways, computational models can play an important role in directing research effort (for example, planning a field campaign) and improving our characterization of the environmental implications of contaminant discharges at systems scales.

\footnotetext{
Methods

Numerical model formulation and simulation. To quantify the potential impact of product-to-parent reversion on TBA metabolite persistence, we conducted numerical simulations of $17 \alpha-\mathrm{TBOH}$ transport and fate in a stream-hyporheic system and compared cases with and without-reversion. The dominant transport processes simulated are advection, dispersion and transient storage in the hyporheic zone ${ }^{24}$. Key assumptions of this model include a well-mixed stream and hyporheic zone at each spatial step, an exponential residence time distribution in the hyporheic zone, and no down-stream transport in the hyporheic zone. We constructed a modified version of this model to represent two interacting species, similar to recent work for the Resazurin-Resorufin tracer ${ }^{25}$. Our 1-D transient storage model simulates transport and fate of $17 \alpha-\mathrm{TBOH}$ and its primary photoproduct, hydroxy-17 $\alpha$-TBOH. A version of the computer code use for simulation may be obtained by contacting the corresponding author.

For our simulations, we selected physical parameters to represent Fourmile Creek near Ankeny, Iowa, USA, a USGS Field Laboratory for the study of Contaminants of Emerging Concern ${ }^{26}$. The physical system is representative of a third order stream in the Midwestern United States. We assumed a wide, rectangular, plane-bed stream with constant discharge of $500 \mathrm{ls}^{-1}$ (average annual average discharge 535 to $4,989 \mathrm{ls}^{-1}$ for water years 2007-2013 at USGS gauge
}

05485605 ) and longitudinal slope of $0.1 \%$ (average gradient for the first $3.1 \mathrm{~km}$ downstream of the USGS gauge site on Fourmile Creek), Darcy-Weisbach friction factor of 0.05 . We assigned longitudinal dispersion at $10^{-8} \mathrm{~m}^{2} \mathrm{~s}^{-1}$. We used a 4-point implicit numerical solution scheme to iterate to a steady-state normal depth and velocity for the network ${ }^{27}$. We specified a hyporheic cross-sectional area of $1 \mathrm{~m}^{2}$, compared with a stream cross-sectional area of $0.68 \mathrm{~m}^{2}(2 \mathrm{~m}$ width, $0.34 \mathrm{~m}$ depth). This ratio of hyporheic to stream area (commonly $A_{\mathrm{s}} / A$ ) is 1.5 . We applied a hyporheic exchange rate of $0.036 \mathrm{~h}^{-1}\left(10^{-5} \mathrm{~s}^{-1}\right)$. Both hyporheic area and exchange rate are within typically observed ranges for studies using the transient storage model $^{28}$.

We simulated a constant-rate input of $5 \mathrm{ngl}^{-1} 17 \alpha-\mathrm{TBOH}$ at the upstream end of the model domain into the stream. This loading is representative of a low concentration, sustained input due to, for example, leaching from field-applied manure to an agricultural landscape or a leaky manure storage pond. Forward and reversion rate constants were estimated using photolysis and reversion data presented in Qu et al. ${ }^{1}$ The reversion rate from hydroxy-17 $\alpha-\mathrm{TBOH}$ to $17 \alpha-\mathrm{TBOH}$ was assumed constant in both the stream and hyporheic zone with a rate constant of $0.02 \mathrm{~h}^{-1}$. The peak photodegradation rate constant for $17 \alpha-\mathrm{TBOH}$ was simulated as $2 \mathrm{~h}^{-1}$. No photodegradation was assigned to hydroxy-17 $\alpha-\mathrm{TBOH}$ The photodegradation rate was simulated as time-dependent, using a half sinewave to assign a value during a $12 \mathrm{~h}$ photoperiod (06:00 to 18:00) and the rate set to zero outside of the photoperiod ${ }^{29}$. We assumed a constant photodegradation rate through the well-mixed water column, given the shallow stream depths simulated. In other systems light penetration through the water column may be controlled by suspended solids concentrations or changes in solar loading in space (for example, shading) and through time (for example, cloud cover).

On the basis of observations in Qu et al. ${ }^{1}$, photolysis of $17 \alpha-\mathrm{TBOH}$ was assumed to yield $90 \%$ revertible products (that is, hydroxy-17 $\alpha-\mathrm{TBOH}$ ) and $10 \%$ other products not subject to reversion and assumed non-bioactive (for example, species with a substantially modified structure, such as the 11,12-dialdehyde product we previously reported ${ }^{15}$ ). All other parameters were assumed constant in space and time to isolate the effect of the reversion process on results. The model was run continuously until a 24-hour steady-state oscillation was achieved, determined by a change in 24 -h peak values of less than $1 \%$ between subsequent 24-h cycles at all spatial locations. This spin-up period was at least $480 \mathrm{~h}$ for all simulations. Daily maximum and minimum values were extracted from a 24 -hour period at this dynamic steady-state. Daily average values were calculated as the arithmetic average of a 24-hour period at this dynamic steady-state. Model variants were completed with- and without-reversion. The model was solved using a Crank-Nicholson solution scheme with spatial steps of $100 \mathrm{~m}$ over a $200 \mathrm{~km}$ domain and temporal steps of $60 \mathrm{~s}$, after Runkel ${ }^{30}$.

Our model represents an improvement in the representation of physical and chemical processes compared with the de facto baseline model used for regulation of $17 \alpha-\mathrm{TBOH}$ (photolysis in the stream water only; no hyporheic exchange; no product-to-parent reversion). The model includes product-to-parent reversion in both the stream and hyporheic zone, and time-variable photolysis in the stream. Other attenuation processes (for example, sorption, biodegradation) were not simulated because sufficient data are not available to characterize these processes for $17 \alpha-\mathrm{TBOH}$ and its major photoproduct, hydroxy-17 $\alpha$-TBOH. As such, this model represents a system where these attenuation processes are minimized, a worst-case scenario for risk from TBA metabolites. While this model increases the realism of the suite of interacting processes that control the transport and fate of TBA metabolites in the environment, we acknowledge that the assumptions of spatially homogeneous reaction rates and omission of competing attenuation pathways (for example, sorption-desorption, biodegradation, dilution) are limitations of this framework. At this time, there are no published studies characterizing pathways for hydroxy-17 $\alpha-\mathrm{TBOH}$ other than photolysis. We included only processes for which rate constants and controls are characterized in the literature for both $17 \alpha-\mathrm{TBOH}$ and hydroxy-17 $\alpha-\mathrm{TBOH}$.

Published partitioning coefficients for $17 \alpha-\mathrm{TBOH}$ include linear distribution coefficient $\left(K_{\mathrm{d}} ; 2.2-41.1 \mathrm{~kg}^{-1}\right)$ with positive relationships with organic content, $\mathrm{pH}$, clay fraction and cation exchange capacity of the soil ${ }^{31-33}$. Additional available partitioning coefficients include organic carbon normalized $\left(\log K_{\mathrm{oc}} ; 2.77 \pm 0.12\right)$, Octanol-water $\left(\log K_{\text {oww }} ; 2.72 \pm 0.02\right)$, Hexane-water $\left(\log K_{\mathrm{hw}} ;-0.114 \pm 0.006\right)$, chlorophyll-water $\left(\log K_{\text {chw }} ; 3.36 \pm 0.01\right)$, cyclohexane-water $\left(\log K_{\text {cyww }} ; 0.39 \pm 0.07\right)$ and toluene-water $\left(\log K_{\mathrm{tw}} ; 1.987 \pm 0.01\right)$ partitioning coefficients ${ }^{31,33}$. First-order biodegradation rates for $17 \alpha-\mathrm{TBOH}$ were recently reported as $0.0034 \mathrm{~h}^{-1}$ for $5^{\circ} \mathrm{C}$ and 0.0071 to $0.013 \mathrm{~h}^{-1}$ for $20^{\circ} \mathrm{C}$ in aerobic batch microcosms ${ }^{31}$. Using these values, we calculate a $\theta$ for the Arrhenius equation ranging from 1.038 to 1.072 . For typical stream temperatures of $18{ }^{\circ} \mathrm{C}$, biodegradation rate constants are $0.0066-$ $0.011 \mathrm{~h}^{-1}(0.33-0.58 \%$ of the peak forward reaction rate; $33-58 \%$ of the reversion rate). Assuming hyporheic temperatures of $12{ }^{\circ} \mathrm{C}$, biodegradation rate constants are $0.0053-0.0077 \mathrm{~h}^{-1}(0.26-0.38 \%$ of the peak forward reaction rate; $26-38 \%$ of the reversion rate). These values demonstrate the potential rates of other processes controlling $17 \alpha-\mathrm{TBOH}$ transport and fate, but are limited in their applicability to our system. These rates were derived from aerobic mesocosms and we do not expect them to be applicable in the typically anaerobic hyporheic zones. To our knowledge, no further data are available about biotransformation rate dependencies on $\mathrm{pH}$, redox conditions, nor other controls for $17 \alpha-\mathrm{TBOH}$. Our model omits any inflows and outflows of water along the domain. Evapotranspiration from the stream could enrich concentrations, though we expect 
this to be minimal compared with down-stream flux of water in the stream. Finally, we note that gross losses of water from the system to regional groundwater would not effect in-stream concentrations, but would reduce the mass flux of $17 \alpha-\mathrm{TBOH}$ and hydroxy-17 $\alpha$-TBOH through the stream and hyporheic zone.

Environmental samples of $17 \alpha-\mathrm{TBOH}$ and hydroxy-17 $\alpha$-TBOH for model validation are not currently available. Samples collected before discovery of the product-to-parent reversion pathway are extremely limited in their usefulness because interconversion occurs constantly during sample collection and processing, resulting in limited ability to draw meaningful conclusions about field concentrations of $17 \alpha-\mathrm{TBOH}$ and hydroxy-17 $\alpha$-TBOH. Indeed, standardized methods to analyse trace concentrations in complex matrices when looking for hydroxylated products and accounting for or eliminating artifacts from reversion do not currently exist. Furthermore, sampling is complicated by the continuous interaction of multiple species between sample collection and processes; transport and storage in the dark would be subject to reversion, while sample prep in the light (as on a laboratory bench) would be subject to photolysis.

Calculation of JBA for representative cases. To assess the influence of reversion on total bioactivity in the network, we apply a conceptual framework where JBA equals the sum of relative potency multiplied by parent or product concentrations, calculated as:

$$
J B A=B_{17 \alpha} C_{17 \alpha}+B_{\mathrm{OH}} C_{\mathrm{OH}}
$$

where $B$ is a bioactivity coefficient describing the relative bioactivity of each compound compared with a baseline, (bioactivity per concentration) assumed here as $17 \alpha-\mathrm{TBOH}$, and $\mathrm{C}$ is the simulated $24-\mathrm{h}$ mean concentration of each compound normalized to the input concentration of $17 \alpha-\mathrm{TBOH}$. In this study, we use the subscripts $17 \alpha$ for the parent $(17 \alpha-\mathrm{TBOH})$, and $\mathrm{OH}$ for the product (hydroxy$17 \alpha-\mathrm{TBOH})$. This framework is similar to that used for oestrogenic compounds, where oestrogenicity is commonly reported as additive $17 \beta$-estradiol equivalents ${ }^{34}$. At this time, no standard scale akin to oestrogenicity is yet established for androgens, nor are compound-specific bioactivities widely calculated for such compounds. In our study, we consider the de facto regulatory assumption as a baseline case (Case 1; $B_{17 \alpha}=1 ; B_{O H}=0$ ). As relative bioactivities are yet unquantified for TBA metabolites, and to extend our results to other compounds with reversible transformation, we consider four additional cases: Case 2 parent is more bioactive than product (for example, $17 \alpha-\mathrm{TBOH}$ and hydroxy-17 $\alpha-\mathrm{TBOH}$; $\left.B_{17 \alpha}=1 ; B_{O H}=0.3\right)$; Case 3 equal bioactivity of parent and product $\left(B_{17 \alpha}=1\right.$; $B_{\mathrm{OH}}=1$ ); Case 4 product is more bioactive than parent (for example, reduction of estrone to $17 \beta$-estradiol or deconjugation of glucuronides to free steroids; $\left.B_{17 \alpha}=0.3 ; B_{\mathrm{OH}}=1\right)^{35}$ and Case 5 only the product is bioactive $\left(B_{17 \alpha}=0 ; B_{\mathrm{OH}}=1\right)$. These calculations are designed to explore downstream bioactivity exposure as a function of the relative bioactivity of identifiable compounds, one of the emerging cases that must be considered in risk management for compounds' bioactive products.

\section{References}

1. Qu, S. et al. Product-to-parent reversion of trenbolone: unrecognized risks for endocrine disruption. Science 342, 347-351 (2013).

2. Gill, D. R. \& Trapp, J. N. In: Symposium: Impact of implants on performance and carcass value of beef cattle. Publ. P-957, 167-181 (Oklahoma State University, 1997).

3. Jones, G. D., Benchetler, P. V., Tate, K. W. \& Kolodziej, E. P. Mass balance approaches to characterizing the leaching potential of trenbolone acetate metabolites in agro-ecosystems. Environ. Sci. Technol. 48, 3715-3723 (2014).

4. Schiffer, B., Daxenberger, A., Meyer, K. \& Meyer, H. H. D. The fate of trenbolone acetate and melengestrol acetate after application as growth promoters in cattle: Environmental studies. Environ. Health Perspect. 109, 1145-1151 (2001).

5. Cole, E. A. et al. Rates and product identification for Trenbolone acetate metabolite biotransformation under aerobic conditions. Environ. Toxicol. Chem. doi:10.1002/etc.2962 (in the press).

6. Webster, J. P. et al. Occurrence of trenbolone acetate metabolites in simulated confined animal feeding operation (CAFO) runoff. Environ. Sci. Technol. 46, 3803-3810 (2012).

7. Leet, J.K. et al. Assessing impacts of land-applied manure from concentrated animal feeding operations on fish populations and communities. Environ. Sci. Technol. 46, 13440-13447 (2012).

8. Gall, H.E., Sassman, S.A., Lee, L.S. \& Jafvert, C.T. Hormone discharges from a Midwest tile-drained agroecosystem receiving animal wastes. Environ. Sci. Technol. 45, 8755-8764 (2011).

9. Khan, B. \& Lee, L.S. Estrogens and synthetic androgens in manure slury from trenbolone acetate/estradiol implanted cattle and in waste-receiving lagoons used for irrivation. Chemosphere 89, 1443-1449 (2012).

10. Bartelt-Hunt, S. L. et al. Effect of growth promotants on the occurrence of endogenous and synthetic steroid hormones on feedlot soils and runoff from beef cattle feeding operations. Environ. Sci. Technol. 46, 1352-1360 (2012).
11. Ankley, G. T. et al. Effects of the androgenic growth promoter 17- $\beta$-trenbolone on fecundity and reproductive endocrinology of the fathead minnow. Environ. Toxicol. Chem. 22, 1350-1360 (2003).

12. Jensen, K. M., Makynen, E. A., Kahl, M. D. \& Ankley, G. T. Effects of the feedlot contaminant $17 \alpha$-trenbolone on reproductive endocrinology of the fathead minnow. Environ. Sci. Technol. 40, 3112-3117 (2006).

13. Morthorst, J. E., Holbech, H. \& Bjerregaard, P. Trenbolone causes irreversible masculinization of zebrafish at environmentally relevant concentrations. Aquat. Toxicol. 98, 336-343 (2010).

14. Syntex Animal Health, "Synovex Plus (Trenbolone Acetate and Estradiol Benzoate) Implant Environmental Assessment" (1995; Available at http:// www.fda.gov/ucm/groups/fdagov-public/@fdagov-av-gen/documents/ document/ucm072347.pdf.

15. Kolodziej, E. P. et al. Identification and environmental implications of phototransformation products of trenbolone acetate metabolites. Environ. Sci. Technol. 47, 5031-5041 (2013).

16. Fenner, K., Scheringer, M. \& Hungerbühler, K. Persistence of parent compounds and transformation products in a level IV multimedia model. Environ. Sci. Technol. 34, 3809-3817 (2000).

17. Fenner, K., Scheringer, M. \& Hungerbühler, K. Joint persistence of transformation products in chemicals assessment: Case studies and uncertainty analysis. Risk Anal. 23, 35-53 (2003).

18. Gasser, L., Fenner, K. \& Scheringer, M. Indicators for the exposure assessment of transformation products of micropollutants. Environ. Sci. Technol. 41, 2445-2451 (2007).

19. Gross-Sorkin, M. Y., Roast, S. D. \& Brighty, G. C. Assessment of feminization of male fish in English rivers by the environment agency of England and Wales. Environ. Health Perspect. 114, 147-151 (2006).

20. Fenner, K., Kooijman, C., Scheringer, M. \& Hungerbühler, K. Including transformation products into the risk assessment for chemicals: The case of nonylphenol ethoxylate usage in Switzerland. Environ. Sci. Technol. 36, 1147-1154 (2002).

21. Ankley, G.T. et al. Adverse outcome pathways: a conceptual framework to support ecotoxicology research and risk assessment. Environ. Toxicol. Chem. 29, 730-741 (2010)

22. Ng, C.A., Scheringer, M., Fenner, K. \& Hungerbühler, K. A framework for evaluating the contribution of transformation products to chemical persistence in the environment. Environ. Sci. Technol. 45, 111-117 (2011).

23. Cwiertny, D. M., Snyder, S. A., Schlenk, D. \& Kolodziej, E. P. Environment designer drugs: When transformation may not eliminate risk. Environ. Sci. Technol. 48, 11737-11745 (2014).

24. Bencala, K. E. \& Walters, R. A. Simulation of solute transport in a mountain pool-and-riffle stream: a transient storage model. Water Resour. Res. 19, 718-724 (1983).

25. Haggerty, R., Marti, E., Argerich, A., von Schiller, D. \& Grimm, N. B. Resazurin as a "smart" tracer for quantifying metabolically active transient storage in stream ecosystems. J. Geophys. Res. 114, G03014 (2009).

26. Schnoebelen, D. J. et al. Research plan and preliminary results - a field research site for emerging contaminants in Iowa. J. Iowa Acad. Sci. 113, 1-9 (2006).

27. Chen, Y. Water and sediment routing in rivers. In: Modeling of Rivers (ed. Shen, H. J.) 10.12-10.97 (Wiley \& Sons, 1979).

28. Harvey, J.W. \& Wagner, B.J. Quantifying hydrologic interactions between streams and their subsurface hyporheic zones. In Streams and Ground Waters Jones, J. A. \& Mulholland, P. J. (eds). Academic Press: San Diego, USA; $3-44,(2000)$.

29. Chapra, S. C. Surface water-quality modeling (Waveland press, 2008).

30. Runkel, R.L. One-dimensional transport with inflow and storage (OTIS): a solute transport model for streams and rivers. US Geol. Surv. Water Res. Invest. Rep. 98-4018, 73 (1998).

31. Khan, B., Qiao, X. \& Lee, L. S. Stereoselective sorption by agricultural soils and liquid - liquid partitioning of trenbolone $(17 \alpha$ and $17 \beta)$ and trendione. Environ. Sci. Technol. 43, 8827-8833 (2009).

32. Card, M. L., Chin, Y. P., Lee, L. S. \& Khan, B. Prediction and experimental evaluation of soil sorption by natural hormones and hormone mimics. J. Agr. Food Chem. 60, 1480-1487 (2012).

33. Qiao, X., Carmosini, N., Li, F. \& Lee, L. S. Probing the primary mechanisms affecting the environmental distribution of estrogen and androgen isomers. Environ. Sci. Technol. 45, 3989-3995 (2011).

34. Young, W.F. et al.Proposed Predicted-No-Effect Concentrations (PNECs) for Natural and Synthetic Steroid Estrogens in Surface Waters. U.K. Environment Agency. R\&D Technical Report P2- T04/1 (2002).

35. Mansell, D.S. et al. Fate of endogenous steroid hormones in steer feedlots under simulated rainfall-induced runoff. Environ. Sci. Technol. 45, 8811-8818 (2011).

\section{Acknowledgements}

This work was financially supported by the National Science Foundation (NSF) Grant No. CBET-1335711, NSF Grant No. EAR-1331906 for the Critical Zone Observatory for 
Intensively Managed Landscapes (IML-CZO), a multi-institutional collaborative effort and the U.S Department of Agriculture (USDA Grant No. 2013-67019-21365). Any opinions, findings, conclusions, or recommendations expressed here are those of the authors and do not necessarily represent the official views of sponsoring agencies.

\section{Author contributions}

A.S.W., D.M.C. and E.P.K. designed this study. Derivation and numerical solution of the model was completed by A.S.W. Important concentration and rate information were provided by D.M.C. and E.P.K. The numerical experiments and preliminary data analyses were performed by C.C.B. Final data analysis and manuscript writing were primarily completed by A.S.W., with contributions from all authors.

\section{Additional information}

Supplementary Information accompanies this paper at http://www.nature.com/ naturecommunications

Competing financial interests: The authors declare no competing financial interests.

Reprints and permission information is available online at http://npg.nature.com/ reprintsandpermissions/

How to cite this article: Ward, A.S. et al. Coupled reversion and stream-hyporheic exchange processes increase environmental persistence of trenbolone metabolites. Nat. Commun. 6:7067 doi: 10.1038/ncomms8067 (2015). 